Márcia Cristina Aquino Teixeira

Regilene de Jesus Santos - Romina Barreto Sampaio

Lain Pontes-de-Carvalho - Washington L.C. dos-Santos

\title{
A simple and reproducible method to obtain large numbers of axenic amastigotes of different Leishmania species
}

Received: 17 April 2002 / Accepted: 14 May 2002 / Published online: 6 July 2002

(C) Springer-Verlag 2002

\begin{abstract}
This work describes a simple method to yield large amounts of Leishmania amastigote-like forms in axenic cultures using promastigotes as the starting population. The method described induced extracellular amastigote transformation of Leishmania amazonensis (97\%), Leishmania braziliensis (98\%) and Leishmania chagasi $(90 \%)$. The rounded parasites obtained in axenic cultures were morphologically similar, even at the ultrastructural level, to intracellular amastigotes. Moreover, the axenic amastigotes remained viable as measured by their ability to revert back to promastigotes and to infect $\mathrm{BALB} / \mathrm{c}$ mice. L. amazonensis and L. braziliensis promastigotes and axenic amastigotes differed in terms of their Western blot profiles. A $46 \mathrm{kDa}$ protein was recognized by specific antibodies only in axenic and lesion-derived L. amazonensis amastigotes and not in promastigotes.
\end{abstract}

\section{Introduction}

Protozoa of the genus Leishmania display two distinct morphological and three distinct physiological forms. The promastigotes, the flagellated stages, infect the sand fly vector both as a multiplying and also as a mammalinfective form (Sacks 1992) whereas the non-flagellated

M.C.A. Teixeira - R. de Jesus Santos - R.B. Sampaio

L. Pontes-de-Carvalho · W.L.C. dos-Santos $(\bowtie)$

Centro de Pesquisas Gonçalo Moniz, Fundação Osvaldo Cruz,

Av. Waldemar Falcão 121, 40295-001, Salvador, Brazil

E-mail: wluis@cpunet.com.br

Tel.: + 55-71-3568781

Fax: $+55-71-3562155$

M.C.A. Teixeira

Laboratório Central de Saúde Pública Professor Gonçalo Moniz, Av. Waldemar Falcão 123, 40295-001, Salvador, Brazil

R. de Jesus Santos ' L. Pontes-de-Carvalho - W.L.C. dos-Santos Escola Bahiana de Medicina e Saúde Pública,

Av. Dom João VI 274, 40290-000, Salvador, Brazil amastigote resides in mammalian macrophage phagolysosomes. Both morphological stages are responsible for the pathology in their respective hosts (Chang and Dyer 1976; Schlein et al. 1992). The promastigote stages can be easily cultivated in different types of media (Hendricks et al. 1978; Hart et al. 1981; Jaffe et al. 1984) and are frequently used in scientific studies. In contrast, the difficulty in obtaining large numbers of amastigotes, free from host cell contaminants, has hampered the investigation of their metabolic, biochemical and biological properties (Chang 1980; Hart et al. 1981). Moreover, a study on macrophage receptors for Leishmania demonstrated the presence of host immunoglobulins on the surface of lesion-derived amastigotes (Peters et al. 1995), something that would particularly hinder their use in biological and immunological studies.

The first successful long-term propagation of amastigote-like forms of Leishmania, namely Leishmania pifanoi, in a cell-free medium was reported by Pan (1984). Since then, attempts at the cultivation of amastigotes in cell free media have been carried out by many authors (reviewed in Bates 1993; Pan et al. 1993). Some reports have focused on the in vitro transformation of promastigotes to amastigote-like forms in response to elevated temperature (Hendricks 1978; Hunter et al. 1982; Leon et al. 1995), whereas few authors have described serial axenic cultivation of the intracellular stage (Pan 1984; Al-Bashir et al. 1992; Hodgkinson et al. 1996). The axenic cultivation of amastigotes of Leishmania mexicana has been more effective than that of other species of Leishmania (Bates 1993). Some authors have reported Leishmania donovani amastigote transformation in axenic cultures (Al-Bashir et al. 1992; Saar et al. 1998), whereas there is only one study on the differentiation to axenic amastigotes of Leishmania chagasi, another visceral species (Leon et al. 1995).

In spite of these reports on the axenic cultivation of Leishmania amastigotes, there still remain controversies about the reproducibility and/or timing of parasite transformations. In this paper, a simple and quick method of obtaining up to $4 \times 10^{7}$ axenic amastigotes $/ \mathrm{ml}$ 
of culture is described, providing large amounts of pure and viable parasites. This method may obviate the need for using experimental laboratory animals to obtain amastigotes and the laborious procedures for intracellular parasite purification.

\section{Materials and methods}

\section{Parasites and culture}

The Leishmania species used were isolated from human patients and identified as Leishmania amazonensis (MHOM/BR88/BA-125 Leila strain), Leishmania braziliensis (MHOM/BR/3456) and Leishmania chagasi (MHOM/BR2000/Merivaldo2 strain). They were kept by passaging in golden hamsters. The cultures were grown at $22^{\circ} \mathrm{C}$ in Schneider's Drosophila medium (Sigma, St. Louis, Mo., USA), pH 7.2, supplemented with either $10 \%$ fetal calf serum (FCS; Hyclone Laboratories, Logan, Utah, USA) for L. amazonensis or $20 \%$ FCS for L. braziliensis and L. chagasi. The promastigotes used to initialize amastigote axenic cultures were maintained in cultures for no more than ten passages. Different culture conditions for promastigote transformation in relation to: $\mathrm{pH}(5.4,6.4$ and 7.2$)$, temperature $\left(32^{\circ}, 34^{\circ}, 35^{\circ}\right.$ and $\left.37^{\circ} \mathrm{C}\right)$ and FCS concentration $(2.5 \%, 5 \%, 10 \%$ and $20 \%)$, were tested. To determine growth curves, parasite cultures were initiated at a concentration of $5 \times 10^{6}$ promastigotes $/ \mathrm{ml}$ in $25 \mathrm{~cm}^{2}$ tissue culture flasks containing $5 \mathrm{ml}$ of medium. Cell density was estimated using a haemocytometer. To disrupt the aggregates which were formed mainly in L. braziliensis cultures, parasites were passed through a 25 -gauge needle before counting. Intracellular amastigotes were isolated from footpad lesions of infected hamsters and purified using a Percoll (Sigma) gradient, as previously reported (Chang 1980).

\section{Morphological assessment}

To evaluate the morphology of transformed parasites, axenic amastigotes were harvested from cultures and either smeared onto slides for Giemsa staining or spun down in Eppendorf tubes for electron microscopy. The supernatant was discarded and the pellets were fixed for $1 \mathrm{~h}$ at $4^{\circ} \mathrm{C}$ with $2 \%$ glutaradehyde. Parasites were pelleted, post-fixed with $1 \%$ osmium tetroxide for $30 \mathrm{~min}$ at room temperature, dehydrated in ethanol-propylene oxide series and embedded in Spurr resin. After polymerization, ultrathin sections were collected in copper grids, stained with uranyl acetate and lead citrate, and examined on a Zeiss EM109 electron microscope at $50 \mathrm{kV}$.

\section{Western blot analysis}

Lysates of promastigotes, amastigotes from lesions and axenic amastigotes were subjected to $10 \%$ polyacrylamide gel electrophoresis (Laemmli 1970). Lysates from different forms of the same Leishmania species were run in the same gel and a total of $10^{7}$ lysed parasites of each Leishmania stage was loaded per lane. The separated proteins were analyzed by Western blot as described elsewhere (Balanco et al. 1998), utilizing an 1:200 dilution of a pool of five sera from mice chronically infected with $L$. amazonensis.

\section{Functional studies}

The viability and functional status of amastigote-like forms were tested by assessing their ability to revert back to promastigotes and to infect mice. Reversion to promastigotes was accomplished by washing the parasites three times in $0.15 \mathrm{M}$ phosphate-buffered saline $(\mathrm{pH}$ 7.2) and transferring them to Schneider's medium with $10-20 \% \mathrm{FCS}, \mathrm{pH} 7.2$, at $22^{\circ} \mathrm{C}$. To evaluate infectivity, axenic amastigotes and promastigotes of $L$. braziliensis and L. amazonensis were inoculated into BALB/c mouse hind footpads. The BALB/c mice were obtained from the Gonçalo Moniz Research Center animal facilities, and were maintained under specific pathogen-free conditions, with balanced mouse food and water ad libitum. The infection experiments were conducted in accordance with the Oswaldo Cruz Foundation guidelines for experimentation with animals.

\section{Results}

\section{Cultivation of amastigote-like forms}

The first aim of this study was to monitor parasite growth and transformation in axenic cultures in order to establish the best conditions for obtaining amastigotelike forms. Starting with a $100 \%$ promastigote population and using Schneider's medium, cultures were subjected to variations in $\mathrm{pH}$, temperature and concentration of FCS to obtain the maximal rate of transformation for each Leishmania species. For L. braziliensis, a high proportion $(98 \%)$ of amastigote-like forms were observed in 3-day cultures supplemented with $20 \%$ FCS, at pH 5.4 and carried out at $34^{\circ} \mathrm{C}$ (Fig. 1a). L. amazonensis parasites had similar levels of differentiation into amastigote-like forms $(97 \%)$ by the 7 th to 10th days of culture in Schneider's medium with $5 \%$ FCS, $\mathrm{pH} 5.4$, at $32^{\circ} \mathrm{C}$ (Fig. 1b). For L. chagasi, $90 \%$ of the promastigotes transformed into amastigote-like forms after 13 days of axenic culture in Schneider's with $20 \%$ FCS, pH 7.2 and at $35^{\circ} \mathrm{C}$ (Fig. 1c). Table 1 summarizes the best culture conditions for obtaining amastigote-like forms for each Leishmania species.

\section{Morphology under light and electron microscopy}

Optical microscopy of Giemsa-stained amastigote-like forms of the three Leishmania species studied revealed oval or pyriform cells lacking a free flagellum. Dividing amastigotes with two nuclei and two kinetoplasts could readily be observed in L. amazonensis, less frequently in L. braziliensis and even less frequently in L. chagasi cultures (not shown). The non-motile, oval forms of L. braziliensis from the $3 \mathrm{rd}$ day of axenic culture also had an amastigote morphology at the ultrastrutural level, e.g. a non-emergent flagellum inside the flagellar pocket (Fig. 2).

\section{Viability of axenic amastigotes}

Axenic amastigotes of all three Leishmania species were able to revert back to promastigotes when cultured under suitable conditions. The proportion transforming was high, as shown by the absence or low numbers of either amastigotes or dead cells in L. braziliensis (Fig. 3) and L. amazonensis cultures (less than 10\%), and in L. chagasi cultures (less than $30 \%$, mostly due to the 
presence of dead cells/cell debris). In addition, BALB/c mice could be infected in the hind footpads both with axenic amastigote-derived promastigotes or axenic amastigotes, as L. braziliensis and L. amazonensis could be isolated 2-3 months later from skin lesions and from draining lymph nodes of infected mice. The development
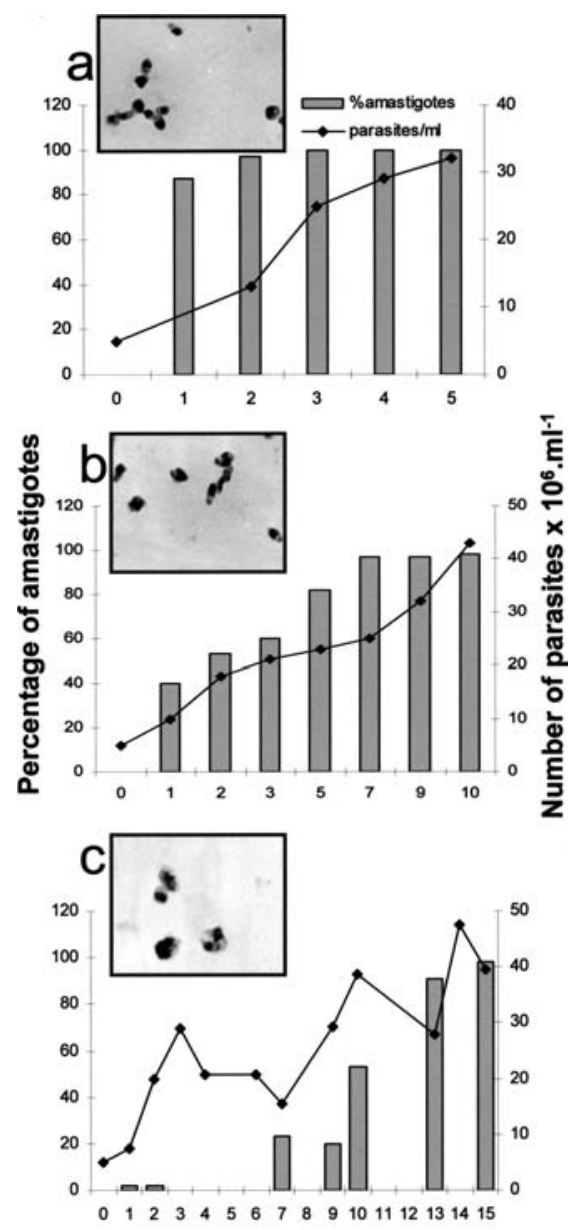

Days of culture

Fig. 1. In vitro growth of Leishmania amastigotes in axenic cultures: a $L$. braziliensis, b $L$. amazonensis and c $L$. chagasi. Cultures were initiated with $5 \times 10^{6}$ promastigotes $/ \mathrm{ml}$ and kept under the specific conditions described in the Materials and methods section. Lozenges represent the number of parasites as determined by counting in a haemocytometer, and bars represent the percentages of amastigote-like forms. Insets show the typical oval- or elliptical-shape axenic amastigotes of each Leishmania species (Giemsa, $\times 4,800$ )

Table 1. Axenic culture conditions, in Schneider's medium, for the transformation of Leishmania promastigotes into amastigote-like organisms

\begin{tabular}{llrrcl}
\hline Species & $\begin{array}{l}\text { Temper- } \\
\text { ature }\end{array}$ & pH & FCS & $\begin{array}{l}\text { Days of } \\
\text { culture }\end{array}$ & $\begin{array}{l}\text { Percent } \\
\text { amastigotes }\end{array}$ \\
\hline L. braziliensis & $34^{\circ} \mathrm{C}$ & 5.4 & $20 \%$ & 3 & $98 \%$ \\
L. amazonensis & $32^{\circ} \mathrm{C}$ & 5.4 & $5 \%$ & 7 & $97 \%$ \\
L. chagasi & $35^{\circ} \mathrm{C}$ & 7.2 & $20 \%$ & 13 & $90 \%$ \\
\hline
\end{tabular}

of lesions in $\mathrm{BALB} / \mathrm{c}$ mice infected with L. amazonensis axenic amastigotes was similar to that observed in promastigote-infected mice (Fig. 4). In fact, two of the three mice infected with axenic amastigotes developed lesions

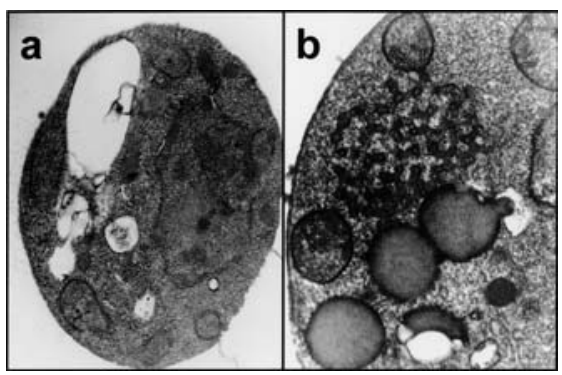

Fig. 2a, b. Electron micrograph of L. braziliensis amastigote-like forms from axenic cultures. L. braziliensis promastigotes were cultivated in Schneider's medium with $20 \%$ of FCS, pH 5.4 and at $34^{\circ} \mathrm{C}$. After 3 days, parasites were harvested from cultures and fixed with glutaraldehyde. Note the non-emergent flagellum inside the flagellar pocket $(\mathbf{a} \times 34,800)$ and lipids droplets in cytoplasm $(\mathbf{b}$ $\times 41,500)$

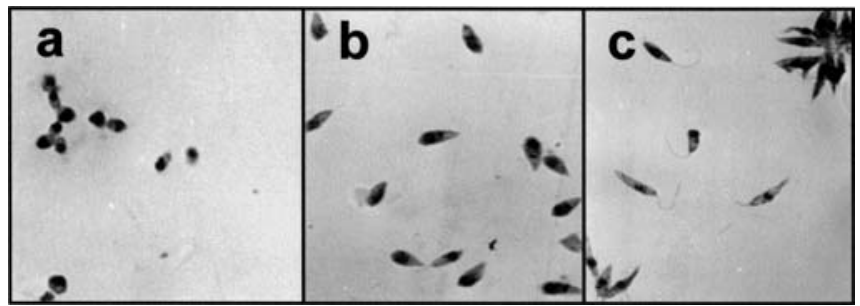

Fig. 3a-c. L. braziliensis amastigote-like forms revert to promastigotes. Axenic amastigotes of L. braziliensis were subjected to suitable conditions for transformation to promastigotes and monitored daily by optical microscopy after staining. a Axenic amastigotes in Schneider's with $20 \%$ FCS at pH 5.4 and $34^{\circ} \mathrm{C}$; b 3-day culture and c 4-day culture in Schneider's medium with $20 \%$ FCS at pH 7.2 and $22^{\circ} \mathrm{C}($ Giemsa, $\times 4,800)$

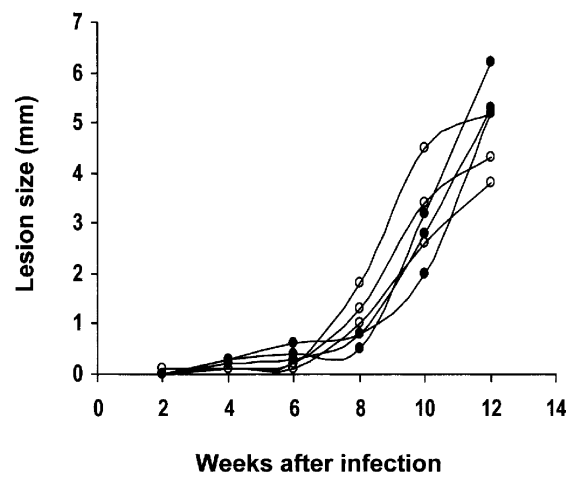

Fig. 4. Infectivity of axenic amastigotes. Groups of three BALB/c mice were infected in hind footpads with either $10^{5}$ axenic amastigotes (closed circles) or $10^{5}$ promastigotes (open circles) of Leishmania amazonensis. Lesion sizes were calculated by subtracting the widths of uninfected from infected footpads. Each curve corresponds to data obtained from an individual animal. Data are representative of two experiments 
slightly larger than those observed in mice infected with promastigotes (Fig. 4).

Stability of axenic amastigotes in culture

The non-motile and ovoid shaped forms of L. amazonensis proliferated in culture and could be inoculated into fresh medium and expanded at least twice. After 3 days, no promastigotes could be detected in L. braziliensis axenic cultures. However, the parasites demonstrated morphological signs of cell injury and started to die by the 5th day of culture. By the 15th day of culture, most of the L. chagasi axenic amastigotes were morphologically unhealthy (they had an irregular cytoplasm and a fragmented nucleus).

Recognition of Leishmania proteins by antiserum

The anti-L. amazonensis serum recognized a $46 \mathrm{kDa}$ protein in $L$. amazonensis amastigotes (axenic and lesion-derived), but not in promastigotes (Fig. 5). At least three bands corresponding to proteins with apparent molecular weights larger than $84 \mathrm{kDa}$ were observed in axenic, but not in lesion L. amazonensis amastigotes. Faint bands between 28 and $32 \mathrm{kDa}$ were revealed only in lesion-derived L. amazonensis amastigotes, whereas a single band of around $30 \mathrm{kDa}$ appeared in both axenic amastigotes and promastigotes. A $65 \mathrm{kDa}$ protein was strongly recognized in L. braziliensis promastigotes and very weakly in axenic amastigotes and bands of 113 and $117 \mathrm{kDa}$ were present only in promastigotes.

\section{Discussion}

In this report, the conditions for the production of large numbers of axenic amastigotes of $L$. amazonensis, L. braziliensis and L. chagasi using Schneider's medium are described. The morphological and ultrastructural characteristics of the axenic amastigotes were very similar to those of lesion amastigotes and their propagation was neither time-consuming nor technically demanding. The amastigote-like forms were viable and expressed stage-specific antigens.

Some authors have accomplished the serial cultivation of Leishmania axenic amastigotes in cell-free medium with a complex composition, including a mixture of nucleotides and vitamins (Pan 1984), or with different protein sources and rabbit blood lysate (Al-Bashir et al. 1992). The diversity of reagents in the prepared media may impede the reproducibility of the results in different laboratories. In fact, we have carefully tested the RBLM medium for axenic amastigotes, as described elsewhere (Al-Bashir et al. 1992), without success for any of the three Leishmania species studied (data not shown).
Specific requirements for temperature, $\mathrm{pH}$ and concentration of FCS were observed for the three species of Leishmania studied, indicating that culture conditions for amastigote differentiation in cell-free media is species dependent. For example, a relatively low $\mathrm{pH}$ (5.4) in the medium was required for the transformation of L. braziliensis and L. amazonensis, but not of L. chagasi. At $\mathrm{pH} 5.4$, the maximum rate of promastigote transformation of this species was $26 \%$ (4-day culture) and the cells died by the 6th day of culture (data not shown). This was in contrast to $90 \%$ transformation at $\mathrm{pH} 7.2$ (13-day culture) under the same conditions. These specific requirements demonstrate differences in $\mathrm{pH}$ and temperature sensitivities among Leishmania species.

Cutaneous and mucocutaneous leishmaniasis in South America are mainly caused by L. braziliensis (Grimaldi et al. 1989). This species differs from others by the presence of small numbers of amastigotes in the lesions, hindering their isolation from tissue. Indeed, this very paucity of $L$. braziliensis amastigotes in skin lesions prevented their purification in large enough numbers to allow their comparison with axenic culture-derived amastigotes in the present study. Therefore, obtaining extracellular L. braziliensis amastigotes forms would facilitate a number of biochemical and immunological studies on this parasite. Eperon and McMahon-Pratt (1989) have previously reported the transformation of L. braziliensis promastigotes to amastigotes in Schneider's medium at $28^{\circ} \mathrm{C}$, without indicating the culture $\mathrm{pH}$. In their study, an acclimatization period and a slow increase in temperature was necessary. Balanco and collaborators (1998) have also obtained L. braziliensis amastigotes in axenic cultures using a different medium (UM-54) at $34^{\circ} \mathrm{C}$ and $\mathrm{pH} 6.3$. With the $2^{\circ} \mathrm{C}$ increments in temperature, 1 week was required to obtain $95 \%$ of amastigote-like forms in culture. As described herein, a rapid and high proportion $(98 \%)$ ) of differentiation of L. braziliensis promastigotes into amastigote-like forms was observed, without changes in $\mathrm{pH}$ or temperature during cultivation.

The culture conditions to obtain axenic amastigotes of $L$. amazonensis described here are an adaptation of those reported by Hodgkinson and collaborators (1996). In order to obtain stable amastigote-like populations, these authors increased the temperature by $2^{\circ} \mathrm{C}$ intervals, followed by decreases of $0.5 \mathrm{pH}$ units down to $\mathrm{pH} 5.0$ and then of $0.2 \mathrm{pH}$ units until $\mathrm{pH} 4.6$ was reached. In the study described here, L. amazonensis differentiation was gradual and took at least 7 days to reach maximal levels at $\mathrm{pH} 5.4$, without any changes in culture conditions.

In order to obtain L. chagasi amastigote forms, the cultivation of promastigotes at $37^{\circ} \mathrm{C}$ and/or at an acidic $\mathrm{pH}$ was first tested. None of these conditions led to parasite transformation or growth. It was necessary to decrease the temperature to $35^{\circ} \mathrm{C}$ and increase the $\mathrm{pH}$ to 7.2 in order to stimulate differentiation which took at least 13 days to reach maximal levels. Dividing amasti- 
gote-like forms were easily observed in L. braziliensis and $L$. amazonensis, but very infrequently in $L$. chagasi axenic cultures. This fact, taken together with the relatively long time (13 days) needed for L. chagasi promastigote differentiation, with a lower proportion (maximal of $90 \%$ ) of axenic amastigotes in comparison with L. amazonensis $(97 \%)$ and L. braziliensis $(98 \%)$ axenic amastigote cultures, and the presence of more cell debris in amastigote-derived promastigote cultures, may indicate that $L$. chagasi is more fastidious than the other two Leishmania species in terms of its requirements for in vitro stage transformation.

The good condition of the axenic culture-derived amastigotes was evaluated by four different criteria. First, all three Leishmania species amastigotes had a regular, smooth morphological appearance. Second, L. amazonensis amastigotes could be subcultured, leading to a progressive increase in the homogeneity of the amastigote-like population, with the absence of promastigotes. Third, the axenic amastigotes of all three species were able to revert back to promastigotes. Fourth, L. braziliensis and L. amazonensis axenic amastigotes kept their viability and infectivity to mice by the time ( 3 and 10 days, respectively) of the maximal ratio of transformation in culture, without signs of cellular death.

Despite their homogeneous morphology, L. amazonensis axenic amastigotes displayed a Western blot profile intermediate between promastigotes and intracellular amastigotes. A protein of $46 \mathrm{kDa}$ from L. amazonensis, recognized by serum from infected BALB/c mice, was present only in the axenic and lesion amastigotes. This protein may correspond to a $46 \mathrm{kDa}$ protein that was detected in Leishmania infantum axenic amastigotes using sera from human beings and dogs with visceral leishmaniasis (Cibrelus 1999), to a $45 \mathrm{kDa}$ band which was recognized in L. donovani using a monoclonal antibody specific to the A-2 multi-gene family of proteins, expressed only in amastigotes (Saar et al. 1998) and/or to a $45 \mathrm{kDa}$ protein of $L$. pifanoi detected with a monoclonal antibody in both axenic and intracellular amastigotes by Pan and collaborators (1993). L. amazonensis proteins with apparent molecular weights of 23 and $30 \mathrm{kDa}$ were recognized in both axenic promastigotes and axenic amastigotes, a fact that may be explained by the presence in amastigote axenic cultures of intermediate forms expressing some proteins of the promastigote stage. In this study, proteins around $30 \mathrm{kDa}$ were recognized by antiserum in all three morphological forms. Colmenares and collaborators (2001) have identified a $L$. pifanoi $31 \mathrm{kDa}$ protein, recognized by an amastigote-specific monoclonal antibody, as a cystein proteinase associated with glycolipids. Either the $30 \mathrm{kDa}$ seen in axenic amastigotes or the $32 \mathrm{kDa}$ protein seen in lesion-derived amastigotes could be that cystein proteinase.

The different methods applied for the characterization of Leishmania molecules, such as Western blot and radioimmunoprecipitation using monoclonal antibodies
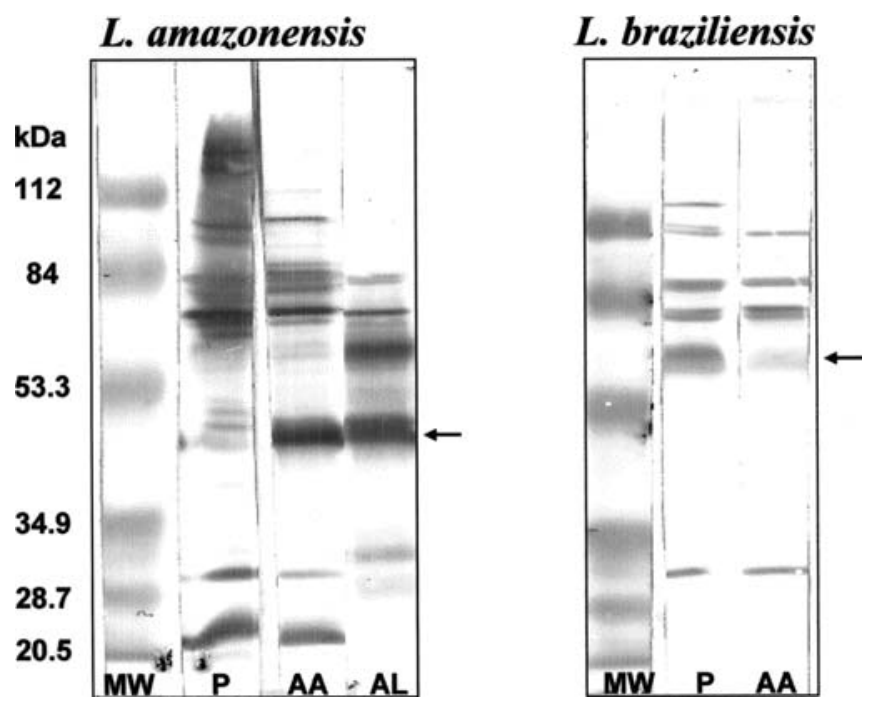

Fig. 5. Reactivity of L. amazonensis and L. braziliensis antigens with polyclonal antibodies. Lysates of promastigotes $(P)$, axenic amastigotes $(A A)$ and amastigotes from lesions $(A L)$ were analysed by Western blot using sera from Leishmania-infected BALB/c mice. The positions of molecular weight $(M W)$ standards are indicated on the left. Arrows indicate the high expression of a $46 \mathrm{kDa}$ protein in L. amazonensis amastigotes and the under expression of a $65 \mathrm{kDa}$ protein in L. braziliensis axenic amastigotes

(Hodgkinson et al. 1996; Pan and McMahon-Pratt 1988), surface radioiodination (Balanco et al. 1997) or Western blot using polyclonal anti-L. amazonensis sera (this report) may account for the distinct group of molecules demonstrated in different studies.

Only L. braziliensis axenic amastigotes and promastigotes were compared in terms of recognition patterns by specific antibodies (Fig. 5b) due to the difficulties in obtaining intracellular amastigotes from the pad lesions of infected hamsters. A protein of $65 \mathrm{kDa}$, which was recognized by specific antibodies more intensely in promastigotes, could indeed be the gp65, and its downregulation in axenic amastigotes shown here is consistent with previously reported data (Kweider et al. 1989). A reduction in intensity of a $117 \mathrm{kDa}$ protein in promastigote-derived amastigotes is also consistent with its down-regulation at this stage.

Axenic amastigotes may be useful for the study of molecules selectively expressed by them and by intracellular amastigotes. The availability of a pure amastigote preparation from different Leishmania species would also facilitate studies aimed at identifying the Leishmania molecules involved in the modulation of chronic infection and/or of potential immunotherapeutic or immunoprophylatic value, as promastigotes enter into contact with the host only for very short periods.

Acknowledgements This work was supported by the Brazilian Ministry of Science and Technology (National Research Council$\mathrm{CNPq}$ and Program for Nucleus of Excellence-PRONEX) and by the Bahia State Government (CADCT). 


\section{References}

Al-Bashir NT, Rassam MB, Al-Rawi BM (1992) Axenic cultivation of amastigotes of Leishmania donovani and Leishmania major and their infectiviy. Ann Trop Med Parasitol 86:487-502

Balanco JMF, Pral EMF, da Silva S, Bijovsky AT, Mortara RA, Alfieri SC (1998) Axenic cultivation and partial characterization of Leishmania braziliensis amastigote-like forms. Parasitology 116:103-113

Bates PA (1993) Axenic culture of Leishmania amastigotes. Parasitol Today 9:143-146

Chang KP (1980) Human cutaneous Leishmania in a mouse macrophage line: propagation and isolation of intracellular parasites. Science 209:1240-1242

Chang KP, Dwyer DM (1976) Multiplication of a human parasite (Leishmania donovani) in phagolysosomes of hamster macrophages in vivo. Science 193:678-80

Cibrelus P, Précigout E, Sereno D, Carcy B, Lemestre JL, Gorenflot A (1999) Secreted antigens of the amastigote and promastigote forms of Leishmania infantum inducing a humoral response in humans and dogs. Parasite 6:121-129

Colmenares M, Tiemeyer M, Kima P, McMahon-Pratt D (2001) Biochemical and biological characterization of the protective Leishmania pifanoi amastigote antigen P-8. Infect Immun 69:6776-6784

Eperon S, McMahon-Pratt D (1989) Extracellular cultivation and morphological characterization of amastigote-like forms of Leishmania panamensis and L. braziliensis. Protozoology 36:502-510

Grimaldi G Jr, Tesh RB, Mcmahon-Pratt D (1989) A review of the geographic distribution and epidemiology of Leishmaniasis in the New World. Am J Trop Med Hyg 41:687-725

Hart DT, Vickerman K, Coombs GH (1981) A quick simple method for purifying Leishmania mexicana amastigotes in large numbers. Parasitology 82:345-355

Hendricks LD, Wood DE, Hajduk ME (1978) Haemoflagellates: commercially available liquid media for rapid cultivation. Parasitology 76:309-316

Hodgkinson VW, Soong L, Duboise SM, McMahon-Pratt D (1996) Leishmania amazonensis: cultivation and characterization of axenic amastigote-like organisms. Exp Parasitol 83:94-105
Hunter KW, Cook CL Hensen SA (1982) Temperature-induced in vitro transformation of Leishmania mexicana. I. Ultrastructural comparison of culture-transformed and intracellular amastigotes. Acta Trop 39:143-150

Jaffe CL, Grimaldi G, McMahon-Pratt D (1984) The cultivation and cloning of Leishmania. In: Morel CM (ed) Genes and antigens of parasites. Fundação Oswaldo Cruz, Rio de Janeiro

Kweider M, Lemesre JL, Santoro F, Kusnierz JP, Sadigursky M, Capron A (1989) Development of metacyclic Leishmania promastigotes is associated with the increasing expression of GP65, the major surface antigen. Parasite Immunol 11:197209

Laemmli UK (1970) Cleavage of structural proteins during assembly of the head of bacteriophage T4. Nature 227:680-685

Leon LL, Soares MJ, Temporal RM (1995) Effects of temperature on promastigotes of several species of Leishmania. J Eukaryot Microbiol 42:219-223

Pan AA (1984) Leishmania mexicana: serial cultivation of intracellular stages in a cell-free medium. Exp Parasitol 58:7280

Pan AA, McMahon-Pratt D (1988) Monoclonal antibodies specific for the amastigote stage of Leishmania pifanoi I. Characterization of antigens associated with stage- and species-specific determinants. J Immunol 140:2406-2414

Pan AA, Duboise SM, Eperon S, Rivas L, Hodgkinson V, Traub-Cseko Y, McMahon-Pratt D (1993) Developmental life cycle of Leishmania-cultivation and characterization of cultured extracellular amastigotes. J Eukaryot Microbiol 40:213-223

Peters C, Aebischer T, Stierhof YD, Fuchs M, Overath P (1995) The role of macrophage receptors in adhesion and uptake of Leishmania mexicana amastigotes. J Cell Sci 108:37153724

Saar Y, Ransford A, Waldman E, Mazareb S, Amin-Spector S, Plumblee J, Turco SJ, Zilberstein D (1998) Characterization of developmentally-regulated activities in axenic amastigotes of Leishmania donovani. Mol Biochem Parasitol 95:9-20

Sacks DL (1992) The structure and function of the surface lipophosphoglycan on different developmental stages of Leishmania promastigotes. Infect Agents Dis 1:200-206

Schlein Y, Jacobson RL, Messer G (1992) Leishmania infections damage the feeding mechanism of the sandfly vector and implement parasite transmission by bite. Proc Nat Acad Sci U S A 89:9944-9948 